\title{
FRW dark energy cosmological model with hybrid expansion law
}

\author{
G. K. Goswami ${ }^{1}$, Anirudh Pradhan ${ }^{2}$, Meena Mishra ${ }^{3}$, A. Beesham ${ }^{4}$ \\ 1,3 Department of Mathematics, Kalyan P. G. College, Bhilai-490 006, C. G., India \\ ${ }^{1}$ Email: gk.goswam9@gmail.com \\ ${ }^{3}$ Email: meenamishra18@gmail.com \\ 2 Department of Mathematics, Institute of Applied Sciences and Humanities, G L A \\ University, Mathura-281 406, Uttar Pradesh, India \\ E-mail: pradhan.anirudh@gmail.com \\ 4 Department of Mathematical Sciences, University of Zululand, Kwa-Dlangezwa 3886, \\ South Africa \\ E-mail: beeshama@unizulu.ac.za
}

\begin{abstract}
In this work, we study a cosmological model of spatially homogeneous and isotropic accelerating universe which exhibits a transition from deceleration to acceleration. For this, Friedmann Robertson Walker(FRW) metric is taken and Hybrid expansion law $a(t)=t^{\alpha} \exp (\beta t)$ is proposed and derived. We consider the universe to be filled with two types of fluids barotropic and dark energy which have variable equations of state. The evolution of dark energy, Hubble, and deceleration parameters etc., have been described in the form of tables and figures. We consider 581 data's of observed values of distance modulus of various SNe Ia type supernovae from union 2.1 compilation to compare our theoretical results with observations and found that model satisfies current observational constraints. We have also calculated the time and redshift at which acceleration in the Universe had commenced.
\end{abstract}

PACS No.: 98.80.Jk; 95.30.Sf

Keywords: FRW universe; SNe Ia data; Observational parameters; Accelerating universe.

\section{Introduction}

The latest findings on observational ground during last three decades by various cosmological missions like observations on type Ia Supernovae (SNe Ia) [1]-[5], CMBR fluctuations [6, 7], large scale structure (LSS) analysis [8, 9], SDSS collaboration [10, 11, WMAP collaboration [12], Chandra X-ray observatory [13], the Hubble space telescope cluster supernova survey $V$ [14], BOSS collaboration[15], the WiggleZ dark energy survey [16] and latest Planck collaboration results [17] all confirms that our universe is undergoing through an accelerating expansion. It is concluded that our universe is dominated by an exotic dark energy (DE). It has negative pressure, so is repulsive and creates acceleration in the universe. Inclusion of cosmological constant in the Einstein's field equation again got importance as a positive cosmological constant is considered as a source of Dark energy. $\Lambda$-CMD cosmology [18, 19] 
is just Eddington-Lemaitre model with the difference that cosmological constant term acts as a source of dark energy with equation of state $p_{\Lambda}=\rho_{\Lambda}=\frac{-\Lambda c^{4}}{8 \pi G}$. However, the model suffers from, inter alia, fine tuning and cosmic coincidence problems [20]. Any acceptable cosmological model must be able to explain the current expansion of the universe.

In any cosmological model, we require to find out rate of the expansion of the universe. Hubble constant determines it. Observationally we require high-precision measurement observatories to estimate Hubble constant. In general relativity, energy conservation equation provides linear relation ship amongst rate of the expansion, pressure, density, and temperature. Dark energy negative pressure and density are also included in it. There is a cosmological equation of state such as the relationship between temperature, pressure, and combined matter energy and vacuum energy density for any region of space. The problem for equation of state for barionic matter has been solved by cosmologists by providing the phases of the universe like stiff matter, radiation dominated and present dust dominated universe, but determination of the equation of state for dark energy is one of the biggest problem in observational cosmology today. Carroll and Hoffman [21] presented a Dark energy (DE) model in which DE is considered in a conventional manner as a fluid by the equation of state (EoS) parameter $\omega_{d e}=\frac{p_{d e}}{\rho_{d e}}$ which is not necessarily constant. We need to investigate equation of state $(\mathrm{EoS})$ parameter for the whole span of the universe. At present, it is nearly equal to -1 . So far two main theories related to variable equation of state for dark energy are quintessence and phantom models of dark energy. In quintessence model $-1 \leq \omega_{d e}<0$ where as in phantom model $\omega_{d e} \leq-1$. Latest surveys such as Supernovae Legacy Survey, Gold sample of Hubble Space Telescope [22, 23], CMB (WMAP, BOOMERANG) [24, 25] and large scale structure (Sloan Digital Sky Survey) data [26] ruled out possibility of $\omega_{d e} \ll-1$ but $\omega_{\text {de }}$ may be little less than -1 . It is found that the present amount of the DE is so small as compared with the fundamental scale (fine-tuning problem). It is comparable with the critical density today (coincidence problem) [18]. So we need a different forms of dynamically changing DE with an effective equation of state $(\mathrm{EoS}), \omega_{(d e)}=p_{(d e)} / \rho_{(d e)}<-1 / 3$. SNe Ia data [27] and a combination of SNe Ia data with CMBR anisotropy and galaxy clustering statistics 9 put limits on $\omega_{(d e)}$ as $-1.67<\omega_{d e}<-0.62$ and $-1.33<\omega_{d e}<-0.79$, respectively.

Komatsu et al. and Hinshaw et al. [26, 28] estimated limits on $\omega_{(d e)}$ as $-1.44<\omega_{(d e)}<$ -0.92 at $68 \%$ confidence level. These observations were based on the combination of cosmological datasets coming from CMB anisotropies, luminosity distances of high redshift type Ia supernovae and galaxy clustering. Recently, Amirhashchi et al. [29, 30], Pradhan et al. [31, Saha et al. [32], Pradhan [33] and Kumar [34] have made study on FRW based dark energy model in which they considered an interacting and non-interacting two type of fluids, one for barotropic matter and other for dark energy.

In this work, we study a particular model which exhibits a transition from deceleration to acceleration. We consider Baryonic matter, dark energy, and "curvature" energy. Both baryonic matter and dark energy have variable equations of state. We study the evolution of the dark energy parameter within a framework of an FRW cosmological model filled with two fluids (barotropic and dark energy). The cosmological implications of this two-fluid scenario are discussed in detail. The model is shown to satisfy current observational constraints. 


\section{Basic field equations and their solutions}

The dynamics of the universe is governed by the Einstein's field equations (EFEs) given by

$$
R_{i j}-\frac{1}{2} R g_{i j}=-\frac{8 \pi G}{c^{4}} T_{i j}
$$

where $R_{i j}$ is the Ricci tensor, $R$ is the scalar curvature, and $T_{i j}$ is the stress-energy tensor taken as $T_{i j}=T_{i j}(m)+T_{i j}(d e)$. We assume that our universe is filled with two types of perfect fluids (since homogeneity and isotropy imply that there is no bulk energy transport), namely an ordinary baryonic fluid and dark energy. The energy-momentum tensors of the contents of the universe are presented as follows: (with the subscripts $m$ and de denoting ordinary matter and dark energy, respectively). $T_{i j}(m)=\left(\rho_{m}+p_{m}\right) u_{i} u_{j}-p_{m} g_{i j}$ and $T_{i j}(d e)=\left(\rho_{d e}+p_{d e}\right) u_{i} u_{j}-p_{d e} g_{i j}$. In standard spherical coordinates $\left(x^{i}=(t, r, \theta, \phi)\right.$, a spatially homogeneous and isotropic Friedmann-Robertson-Walker (FRW) line-element has the form (in units $c=1$ )

$$
d s^{2}=d t^{2}-a(t)^{2}\left[\frac{d r^{2}}{\left(1+k r^{2}\right)}+r^{2}\left(d \theta^{2}+\sin ^{2} \theta d \phi^{2}\right)\right],
$$

Where (i) $k=-1$ is closed universe (ii) $k=1$ is open universe and (iii) $k=0$ is spatially flat universe. Solving EFEs (11) for the FRW metric (2) and energy momentum tensors described above, we get the following equations of dynamic cosmology $2 \frac{\ddot{a}}{a}+H^{2}=-8 \pi G p+\frac{k}{a^{2}}$ and $H^{2}=\frac{8 \pi G}{3} \rho+\frac{k}{a^{2}}$, where $H=\frac{\dot{a}}{a}$ is the Hubble constant. Here an over dot means differentiation with respect to cosmological time $t$. We have deliberately put the curvature term on the right of EFEs, as this term is made to acts like an energy term. For this, we assume that the density and pressure for the curvature energy are as follows $\rho_{k}=\frac{3 k}{8 \pi G a^{2}}, \quad p_{k}=-\frac{k}{8 \pi G a^{2}}$. With this choice, EFEs are read as

$$
2 \frac{\ddot{a}}{a}+H^{2}=-8 \pi G\left(p+p_{k}\right) \& H^{2}=\frac{8 \pi G}{3}\left(\rho+\rho_{k}\right) .
$$

The energy density $\rho$ in is comprised of two types of energy, namely, matter and dark energy $\rho_{m}$ and $\rho_{d e}$ where as the pressure ' $p$ ' is comprised of pressure due to matter and pressure due to dark energy. We can express $\rho=\rho_{m}+\rho_{d e}$, and $p=p_{m}+p_{d e}$.

The energy conservation law(ECL) $T_{; j}^{i j}=0$ provides the following well known equation amongst the density $\rho$, pressure $p$ and Hubble constant $H \dot{\rho}+3 H(p+\rho)=0$, where $\rho=$ $\rho_{m}+\rho_{d e}+\rho_{k}$ and $p=p_{m}+p_{d e}+p_{k}$, are the total density and pressure of the universe, respectively. We see that $\rho_{k}$ and $p_{k}$ satisfy ECL independently, i.e., $\dot{\rho}_{k}+3 H\left(p_{k}+\rho_{k}\right)=0$, so that $\frac{d}{d t}\left(\rho_{m}+\rho_{d e}\right)+3 H\left(p_{m}+p_{d e}+\rho_{m}+\rho_{d e}\right)=0$. We assume that both matter and dark energies are minimally coupled so that they are conserved simultaneously, i.e. $\rho_{m}+3 H\left(p_{m}+\rho_{m}\right)=0$, and $\rho_{d e}+3 H\left(p_{d e}+\rho_{d e}\right)=0$. ECLs are integrable when suitable functions are chosen relating pressure to density: $p_{m}=\omega_{m} \rho_{m}, p_{d e}=\omega_{d e} \rho_{d e}$ and $p_{k}=\omega_{k} \rho_{k}$. In the early part of the universe, it was radiation dominated $\omega_{m}=\frac{1}{3}, \rho_{m} \propto a^{-4}$. Later on when the radiation decoupled from the baryons, the matter dominated era began. In this epoch, $\omega_{m}=0, \rho_{m} \propto a^{-3}$. In order to describe the whole history of the universe through the equation of state, we may write $\rho_{m}=\rho_{\text {dust }}+\rho_{\text {rad }}=\left(\rho_{m}\right)_{0}\left(\frac{a_{0}}{a}\right)^{3}\left[\mu+(1-\mu) \frac{a_{0}}{a}\right]$, where the parameter $\mu$ varies as per the different era of the universe. For a particular era, it is a constant. For example, $\mu=1$ corresponds to the present dust filled era, and $\mu=0$ corresponds to the early era of the universe in which only radiation was present. Here terms with subscript zero are constants 
and describe values at present. Equations of states for energies corresponding to $k$ are as follows $p_{k}=\omega_{k} \rho_{k}$ where $\omega_{k}=-1 / 3$. This gives $\rho_{k} \propto a^{-2}=\left(\rho_{k}\right)_{0}\left[\frac{a_{0}}{a}\right]^{2}$. We use the relationship between scale factor and red shift as $\frac{a_{0}}{a}=1+z$, to write variables in terms of redshift $z$ rather than time. The critical density and the density parameters for energy density, dark energy and curvature density (in units $c=1$ ) are, respectively, defined by

$\rho_{c}=\frac{3 H^{2}}{8 \pi G}, \Omega_{m}=\frac{\rho_{m}}{\rho_{c}}, \Omega_{d e}=\frac{\rho_{d e}}{\rho_{c}}$, and $\Omega_{k}=\frac{\rho_{k}}{\rho_{c}}$. With these in hand, we can write the FRW field equations as follows

$$
H^{2}=H_{0}^{2}\left[\left(\Omega_{m}\right)_{0}\left(\frac{a_{0}}{a}\right)^{3}\left[\mu+(1-\mu) \frac{a_{0}}{a}\right]+\left(\Omega_{k}\right)_{0}\left(\frac{a_{0}}{a}\right)^{2}\right]+H^{2} \Omega_{d e},
$$

and

$$
2 q=1+\frac{3 H_{0}^{2}}{H^{2}}\left[\omega_{m}\left(\Omega_{m}\right)_{0}\left(\frac{a_{0}}{a}\right)^{3}\left[\mu+(1-\mu) \frac{a_{0}}{a}\right]-\frac{1}{3}\left(\Omega_{k}\right)_{0}\left(\frac{a_{0}}{a}\right)^{2}\right]+3 \omega_{d e} \Omega_{d e},
$$

where $q$ is the deceleration parameter defined by $q=-\frac{\ddot{a}}{a H^{2}}$. It is important to mention here that this model represents a decelerating universe in the absence of dark energy. This is so because the deceleration parameter $q$ is positive when the dark energy is zero. We recall

$q \lesseqgtr 1 / 2$ for closed, flat and open universes, respectively. The $\Lambda$ CMD model fits best with the present day observations. In this model, $\Lambda$ accounts for the vacuum energy with its energy density $\rho_{\Lambda}$ and pressure $p_{\Lambda}$ satisfying the equation of state $(\operatorname{EoS}) p_{\Lambda}=-\rho_{\Lambda}=\frac{\Lambda}{8 \pi G} ; \omega_{d e}=-1$. The purpose of this paper is to investigate the effect of $\omega_{d e}$ as a function of time.

We have only two equations and the scale factor ' $a$ ', pressure $p$ and energy density $\rho$ to be determined. So we have to use a certain ansatz. In e print [35], we have developed hybrid expansion law [HEL] for scale factor 'a' as follows $a(t)=t^{\alpha} \exp (\beta t)$, where $\alpha$ and $\beta$ are arbitrary constants. These have been obtained as $\beta=0.0397474 \sim 0.04, \alpha=0.415066 \sim$ 0.415 on the basis of Planck's observational results [17] and the following differential equations obtained from HEL.

$$
\alpha(1+z) H H_{z}=\alpha(q+1) H^{2}=(H-\beta)^{2}=\frac{\alpha^{2}}{t^{2}} .
$$

\section{$2.1 \quad$ Hubble constant $H$}

Eq. (6) is solved for the Hubble constant $H$ as a function of redshift $z$ as follows

$$
(H-\beta)^{\alpha}=A \exp \left(\frac{\alpha \beta}{H-\beta}\right)(1+z),
$$

where constant of integration $\mathrm{A}$ is obtained as $A=0.134$ on the basis of the present value of $H\left(H_{0}=0.07 \mathrm{~Gy}^{-1}\right)$. A numerical solution of Eq. (17) shows that the Hubble constant is an increasing function of red shift.

As it is clear that Hubble constant is in fact not a constant. It varies slowly over red shift and time. Various researchers [15, 16, 36, 37, 38, 39] have estimated values of Hubble constant at different red-shifts using Differential age approach and Galaxy Clustering method. The following table describes various observed values of Hubble constant Hob along with corrections as per red-shift in the range $0 \leq z \leq 2$. We have also calculated and put the corresponding theoretical values of $H$ as per our model in the table. It is found that both observed and theoretical values tally considerably and support our model. 


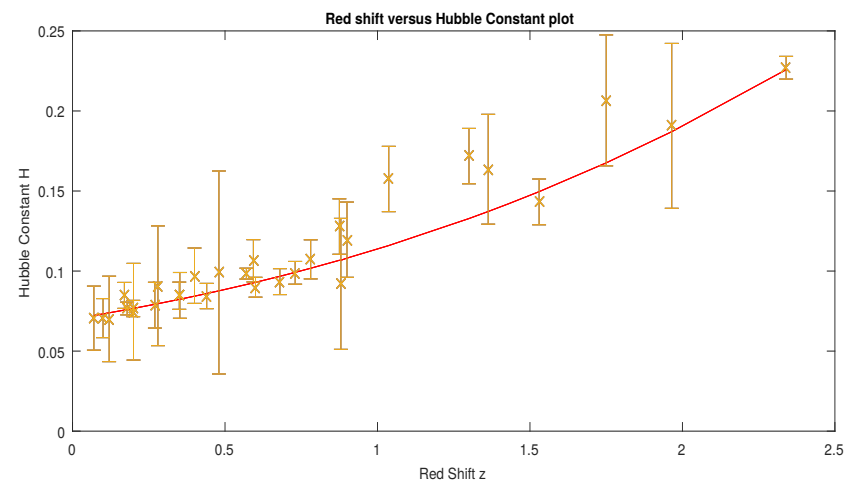

Figure 1: Plot of Hubble constant $(H)$ versus redshift $(z)$

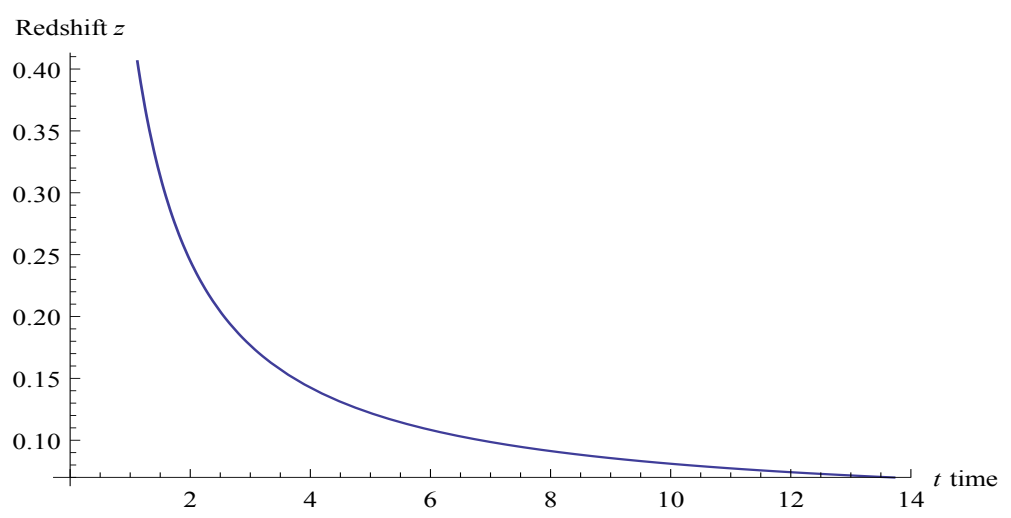

Figure 2: Plot Redshift $(z)$ versus time $(t)$

[Table-1]

(The values of the Hubble constant $(H)$ for redshift $(z)$ ranging between 0 to 2 ) (Hubble constant is expressed in $\mathrm{Gy}^{-1}$ )

\begin{tabular}{|c|c|c|c|c|c|c|}
\hline$z$ & 0.07 & 0.1 & 0.12 & 0.17 & 0.179 & 0.199 \\
\hline Hob & $.0706 \pm .0200$ & $.0706 \pm .0122$ & $.0701 \pm .0267$ & $.0848 \pm .0081$ & $.0766 \pm .0040$ & $.0766 \pm .0051$ \\
\hline Hth & 0.0722454 & 0.0732392 & 0.0739125 & 0.0756344 & 0.0759503 & 0.0766589 \\
\hline \hline$z$ & 0.2 & 0.27 & 0.28 & 0.35 & 0.352 & 0.4 \\
\hline$H o b$ & $.0746 \pm .0302$ & $.0787 \pm .0143$ & $.0908 \pm .0374$ & $.0846 \pm .0085$ & $.0848 \pm .0143$ & $.0971 \pm .0173$ \\
\hline Hth & 0.0766946 & 0.0792498 & 0.0796243 & 0.0823145 & 0.0823932 & 0.0843112 \\
\hline \hline$z$ & 0.44 & 0.48 & 0.57 & 0.593 & 0.60 & 0.68 \\
\hline H0b & $.0844 \pm .0079$ & $.0991 \pm .0634$ & $.0984 \pm .0034$ & $.1064 \pm .0132$ & $.0899 \pm .0062$ & $.0934 \pm .0081$ \\
\hline Hth & 0.0859548 & 0.0876406 & 0.0915919 & 0.0926377 & 0.092959 & 0.0967302 \\
\hline \hline$z$ & 0.73 & 0.781 & 0.875 & 0.88 & 0.90 & 1.037 \\
\hline$H o b$ & $.0989 \pm .0071$ & $0.1073 \pm .0122$ & $0.1278 \pm .0173$ & $.0920 \pm .0409$ & $.1196 \pm .0235$ & $.1575 \pm .0204$ \\
\hline Hth & 0.0991824 & 0.101761 & 0.106723 & 0.106723 & 0.10809 & 0.115938 \\
\hline
\end{tabular}

\begin{tabular}{|c|c|c|c|c|c|c|}
\hline$z$ & 1.3 & 1.363 & 1.43 & 1.53 & 1.75 & 1.965 \\
\hline Hob & $.1718 \pm .0173$ & $.1636 \pm .0343$ & $.1810 \pm .0184$ & $.1432 \pm .0143$ & $.2066 \pm .0409$ & $.1907 \pm .0515$ \\
\hline Hth & 0.132796 & 0.137201 & 0.142047 & 0.149595 & 0.167577 & 0.187053 \\
\hline
\end{tabular}


Figure 1 depicts the variation of the Hubble constant $H$ with red shift $z$. From this figure, we observe that $H$ increases with the increase of red shift. In this figure, cross signs are 31 observed values of Hubble constants $H_{0}$ with corrections where as the linear curve is the theoretical graph of the Hubble constant $H$ as per our model. Figure 2 plots the variation of red shift $z$ with time $t$ which shows that in the early universe the red shift was more than at present.

\subsection{DE parameter $\Omega_{d e}$ and equation of state parameter $\omega_{d e}$ for DE density}

Now, from Eqs.(44), (5) and (66), the density parameter $\Omega_{d e}$ and EoS parameter $\omega_{d e}$ for dark energy are given by

$$
\begin{gathered}
H^{2} \Omega_{d e}=H^{2}-\left(\Omega_{m}\right)_{0} H_{0}^{2}(1+z)^{3}-\left(\Omega_{k}\right)_{0} H_{0}^{2}(1+z)^{2} \\
\omega_{d e}=\frac{(2-3 \alpha) H^{2}-4 \beta H+2 \beta^{2}+\alpha\left(\Omega_{k}\right)_{0} H_{0}^{2}(1+z)^{2}}{3 \alpha\left[H^{2}-H_{0}^{2}\left(\Omega_{m}\right)_{0}(1+z)^{3}-H_{0}^{2}\left(\Omega_{k}\right)_{0}(1+z)^{2}\right]} .
\end{gathered}
$$

Where we have taken $\omega_{m}=0$ and $\mu=1$ for present dust filled universe.

We present the following numerical tables [2] and [3], which display values of the energy parameter $\Omega_{d e}$ and EoS parameter $\omega_{d e}$ for DE versus red shift $z$ ranging between 0 to 1.4.

[Table-2]

(The values of $\Omega_{d e}$ and $z$ ranging between 0 to 3 )

\begin{tabular}{|c|c|c|c|c|c|c|}
\hline$z$ & 0.0 & 0.2 & 0.4 & 0.6 & 0.8 & 1.0 \\
\hline$\Omega_{d e}$ & 0.695 & 0.671884 & 0.609804 & 0.506256 & 0.360992 & 0.176341 \\
\hline
\end{tabular}

[Table-3]

\begin{tabular}{|c|c|c|c|c|c|c|}
\hline 2 & 0 . & 0.1 & 0.2 & 0.3 & 0.4 & \\
\hline$\omega_{c}$ & \begin{tabular}{l|l}
$d e$ & -1.00673 \\
\end{tabular} & -0.961887 & -0.926735 & -0.90137 & -0.886524 & \\
\hline$z$ & 0.5 & 0.6 & 0.7 & 0.8 & 0.9 & 1.0 \\
\hline$\omega_{d e}$ & -0.883867 & -0.896634 & -0.930997 & -0.999402 & -1.13007 & -1.40182 \\
\hline
\end{tabular}

(The values of EoS parameter for DE $\left(\omega_{d e}\right)$ and red shift $(z)$ ranging between 0 to 1 )

Figure 3 depicts the variation of $\Omega_{d e}$ with $z$. Figure 4 plots the variation of $\omega_{d e}$ with $z$. These Figures 3 and 4 support the content of the tables 2 and 3.

It is found that at $z \simeq 1.16321$, there is a singularity while determining $\omega_{d e}$. So we can say that this model works well during $0 \leq z \leq 1.1632$. Moreover, $-1.68889 \leq \omega_{d e} \leq-0.910382$ during $0 \leq z \leq 0.98$. This is the limit on $\omega_{\text {de }}$ found by various surveys. The recent supernovae SNI $997 f f$ at $z \simeq 1.7$ is consistent with a decelerated expansion at the epoch of high emission ( Benitez et al. [40], Turner \& Riess [41]). 


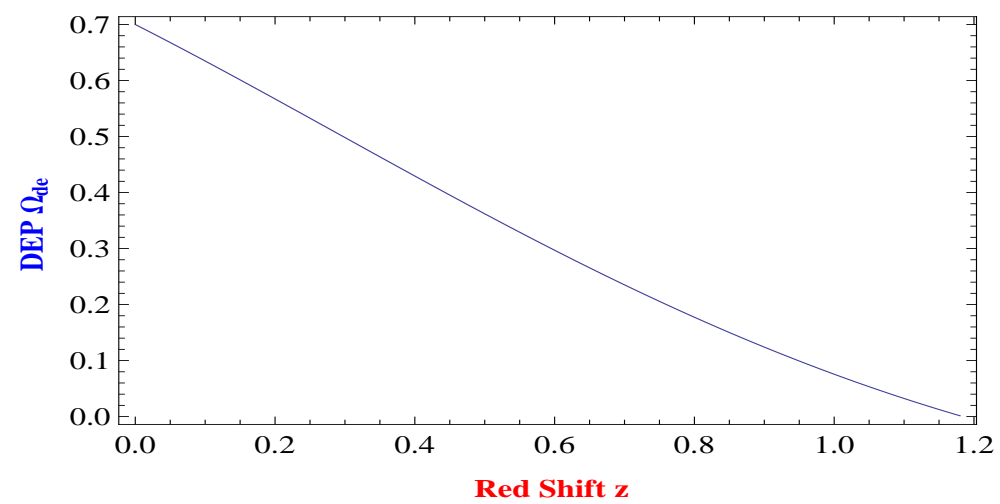

Figure 3: Plot of Energy density parameter for DE $\left(\Omega_{d e}\right)$ versus redshift $(z)$

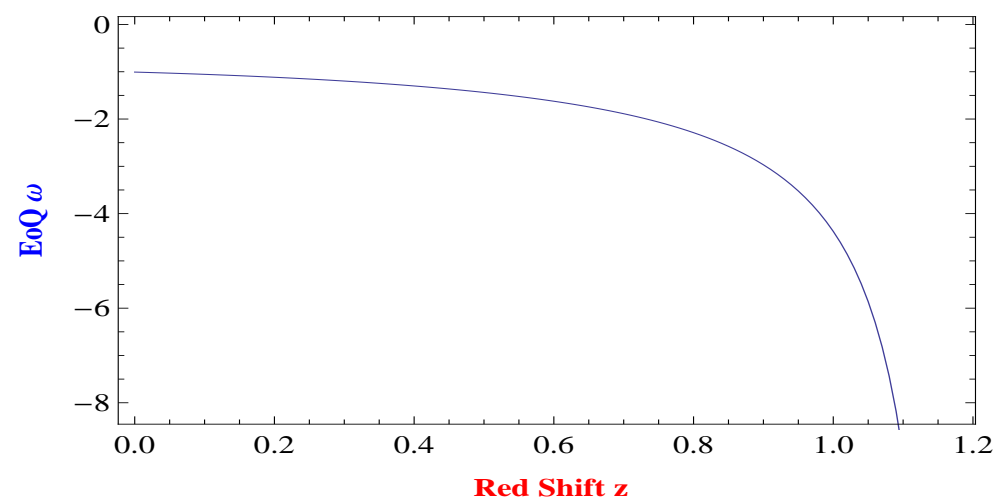

Figure 4: EoS parameter for DE $\left(\omega_{d e}\right)$ versus redshift $(z)$

\subsection{Time at which energy density parameter for $\mathrm{DE} \Omega_{d e}$ opposes deceleration}

From Eqs. (7) and (8), we observe that

$z \rightarrow 1.1633, \quad \Omega_{d e} \rightarrow-0.0000984892$ and when $z \rightarrow 1.1632, \quad \Omega_{d e} \rightarrow 0.0000157204$

It means

$\Omega_{d e} \rightarrow 0$, when $z \rightarrow 1.16325$ i.e. $t \rightarrow 4.91961$ Gyrs.

At this time $q \rightarrow 0.092543$. Thus, as per our model, dark energy begins its role of opposing deceleration and exerting negative pressure at time $t \rightarrow 4.91961$ Gyrs.

\subsection{Densities in our model}

From ECLs for the matter density and dark energy density we obtain: $\rho_{m}=\left(\Omega_{m}\right)_{0}(1+z)^{3}(\mu+$ $(1-\mu)(1+z))\left(\rho_{c}\right)_{0}$ and $\rho_{d e}=\Omega_{d e}\left[\left(\Omega_{m}\right)_{0}[1+z]^{3}[\mu+(1-\mu)(1+z)]+\left(\Omega_{k}\right)_{0}[1+z]^{2}+\Omega_{d e}\right]\left(\rho_{c}\right)_{0}$, where $\left(\rho_{c}\right)_{0}=1.88 \times 10^{-29} \mathrm{gm} / \mathrm{cm}^{3}$. Now we present the following table which describes the dark energy density $\rho_{d e}$ in units of $\left(\rho_{c}\right)_{0}$ for redshift $z$ ranging between 0 and 1 .

[Table-4] 
(The values of energy density for $\operatorname{DE}\left(\rho_{d e}\right)$ for redshift $(z)$ ranging in between 0 and 1 )

\begin{tabular}{|c|c|c|c|c|c|c|}
\hline$z$ & 0.0 & 0.1 & 0.2 & 0.3 & 0.4 \\
\hline$\rho_{d e} /\left(\rho_{c}\right)_{0}$ & 0.695 & 0.752362 & 0.80457 & 0.848396 & 0.879828 \\
\hline$z$ & 0.5 & 0.6 & 0.7 & 0.8 & 0.9 & 1.0 \\
\hline$\rho_{d e} /\left(\rho_{c}\right)_{0}$ & 0.893955 & 0.884863 & 0.845532 & 0.767754 & 0.642085 & 0.45784 \\
\hline
\end{tabular}

\subsection{Transition from deceleration to acceleration}

Now we present the following table which describes the transition from deceleration to acceleration.

[Table-5]

(The values of the deceleration parameter $(q)$ for redshift $(z)$ ranging between 0 and 10 )

\begin{tabular}{|c|c|c|c|c|c|c|c|c|c|}
\hline$z$ & & 0 & 1 & \multicolumn{2}{|l|}{2} & \multicolumn{2}{|l|}{3} & \multicolumn{2}{|c|}{4} \\
\hline$q$ & & 552016 & 0.0253422 & 0.5219 & & \multicolumn{2}{|c|}{0.849706} & \multicolumn{2}{|c|}{1.04866} \\
\hline & $z$ & 5 & 6 & 7 & & 8 & & 9 & 10 \\
\hline & $q$ & 1.16973 & 1.24585 & 1.29563 & & 2943 & & 5318 & 1.37036 \\
\hline
\end{tabular}

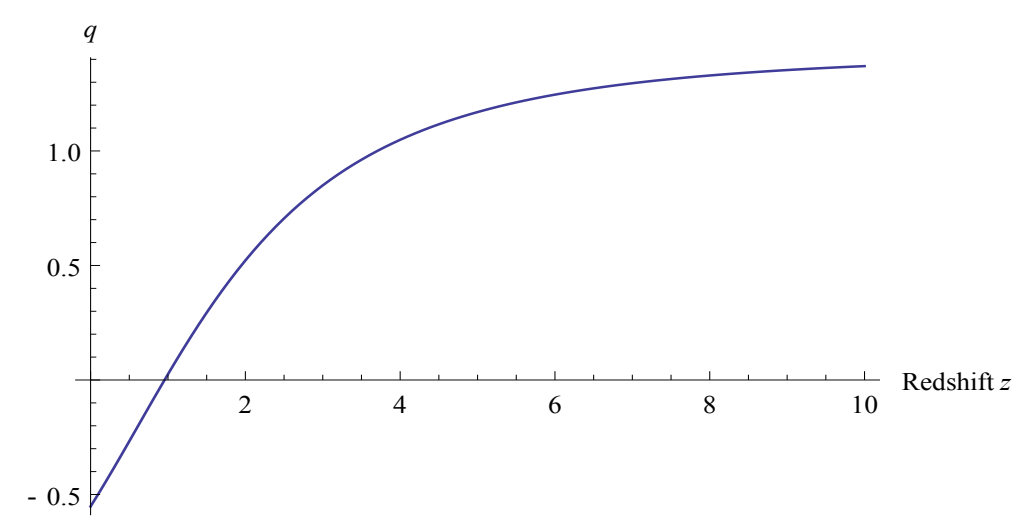

Figure 5: Deceleration parameter $q$ versus red shift $z$

Figure 5 depicts the variation of DP $(q)$ with red shift $z$ based on the above table. The figure describes the fact more clearly.

\subsection{Time at which acceleration had began}

At $z=0.9557, \& 0.9558$, our model gives following values of Hubble constant $H$, deceleration parameter $q$ and and corresponding time.

$$
H(0.9557) \rightarrow 0.111206, \quad q(0.9557) \rightarrow-0.0000124355, \quad t(0.9557) \rightarrow 5.81124,
$$

and

$$
H(0.9558) \rightarrow 0.111212, \quad q(0.9558) \rightarrow 0.0000450098, \quad t(0.9558) \rightarrow 5.81078 .
$$

This means that the acceleration had begun at $z \rightarrow 0.95575, t \rightarrow 5.81104$ Gyr, $H \rightarrow$ 0.111209 Gyr $^{-1}$. At this time $\Omega_{d e}=0.220369$ and $\omega_{d e}=-1.54715$. 


\section{Luminosity distance versus red shift relation}

Red shift-luminosity distance relation [21, 42] ia an important observational tool to study the evolution of the universe. The expression for luminosity distance $\left(D_{L}\right)$ is obtained in term of red shift as the light coming out of a distant luminous body get red shifted due to the expansion of the universe. We determine the flux of a source with the help of luminosity distance. It is given as $D_{L}=a_{0} r(1+z)$, where $\mathrm{r}$ is the radial co ordinate of the source. We consider a ray of light having initially $\frac{d \theta}{d s}=0$ and $\frac{d \phi}{d s}=0$, then geodesic for the metric (5) will determine $\frac{d^{2} \theta}{d s^{2}}=0$ and $\frac{d^{2} \phi}{d s^{2}}=0$. So if we pick up a light ray in a radial direction, then it continues to move along the $r$-direction always, and we get following equation for the path of light $d s^{2}=c^{2} d t^{2}-\frac{a^{2}}{1+k r^{2}} d r^{2}=0$. As we have seen, the effect of curvature is very small at present, $\left(\Omega_{k}\right)_{0}=0.005$, so for the sake of simplification, we take $k=0$. From this we obtain

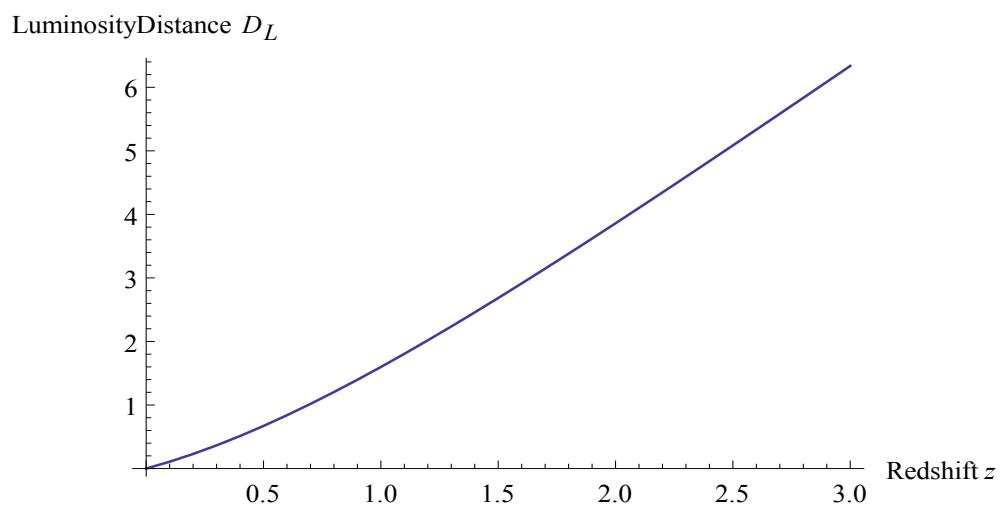

Figure 6: Luminosity distance $\left(D_{L}\right)-$ Redshift $(z)$ Plot

$r=\int_{0}^{r} d r=\int_{0}^{t} \frac{c d t}{a(t)}=\frac{1}{a_{0} H_{0}} \int_{0}^{z} \frac{c d z}{h(z)}$, where we have used $d t=d z / \dot{z}, \dot{z}=-H(1+z)$ and $h(z)=\frac{H}{H_{0}}$. So we get following expression for the luminosity distance

$$
D_{L}=\frac{c(1+z)}{H_{0}} \int_{0}^{z} \frac{d z}{h(z)}
$$

In our earlier work [43, 44, we already obtained luminosity distance.

Solving Eqs. (7) - (91) and (10) numerically, we get following table for values of luminosity distance $\left(D_{L}\right)$ at various red shifts.

[Table-6]

( Luminosity distances $\left(D_{L}\right)$ at redshifts $(z)$ in the range 0 to 3 )

\begin{tabular}{|c|c|c|c|c|c|c|c|c|c|}
\cline { 2 - 10 } \multicolumn{1}{c|}{$z$} & 0.0 & 0.2 & 0.4 & 0.6 & 0.8 & 1.0 & 1.2 & 1.4 \\
\cline { 2 - 10 } \multicolumn{1}{c|}{$D_{L}$} & 0.0 & 0.229565 & 0.512198 & 0.839454 & 1.2038 & 1.59863 & 2.01827 & 2.4579 \\
\hline$z$ & 1.6 & 1.8 & 2.0 & 2.2 & 2.4 & 2.6 & 2.8 & 3.0 \\
\hline$D_{L}$ & 2.9135 & 3.38175 & 3.85993 & 4.34584 & 4.83769 & 5.33408 & 5.83385 & 6.33612 \\
\hline
\end{tabular}

Figure 6 depicts the variation of the luminosity distance with red shift based on Table-6. We observe that the luminosity distance is an increasing function of red shift. 


\section{Distance modulus $\mu$ and apparent magnitude $m_{b}$ for type Ia Supernovas (SNe Ia):}

The distance modulus $\mu$ of a source is defined as $\mu=m_{b}-M$, where $m_{b}$ and $M$ are the apparent and absolute magnitude of the source, respectively. The distance modulus is related to the luminosity distance through the following formula $\mu=M-m_{b}=5 \log _{10}\left(\frac{D_{L}}{M p c}\right)+25$. Type Ia supernova (SN Ia) are standard candle. They have a common absolute magnitude $M$ irrespective of the red shift $z$. We use following equation for luminosity distance for a supernova at very small red shift $D_{L}=\frac{c z}{H_{0}}$.

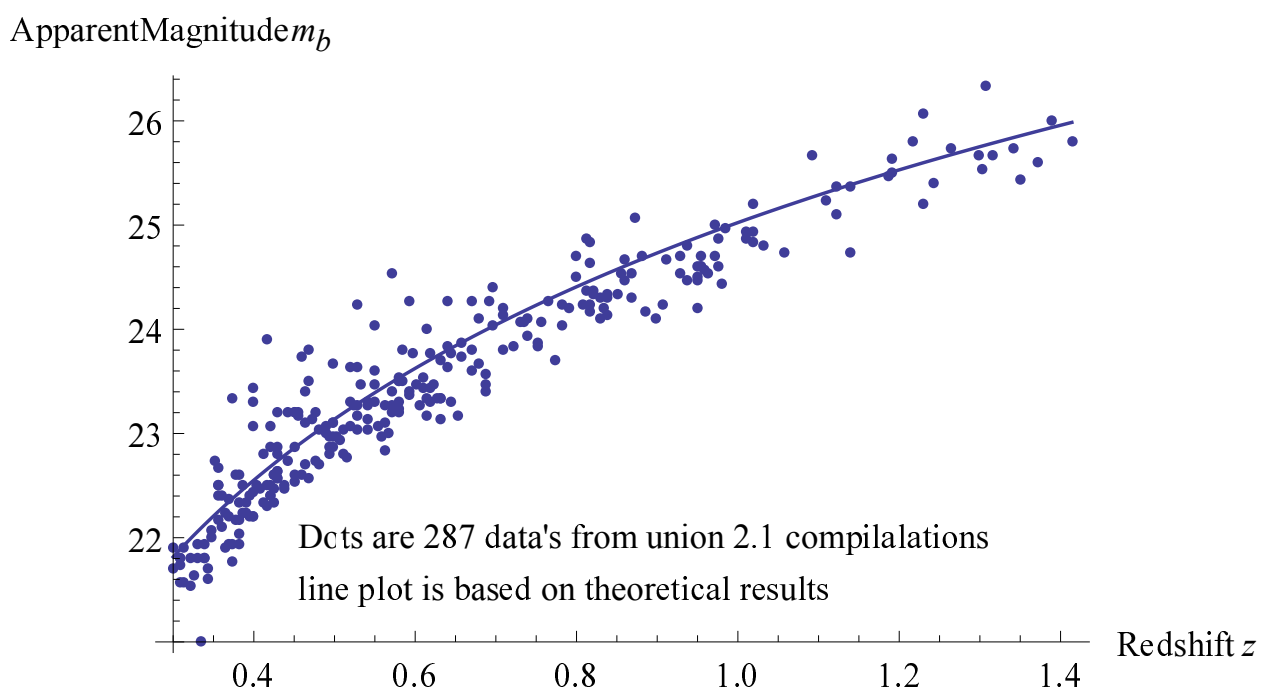

Figure 7: Plot of apparent magnitude $\left(m_{b}\right)$ versus red shift $(z)$

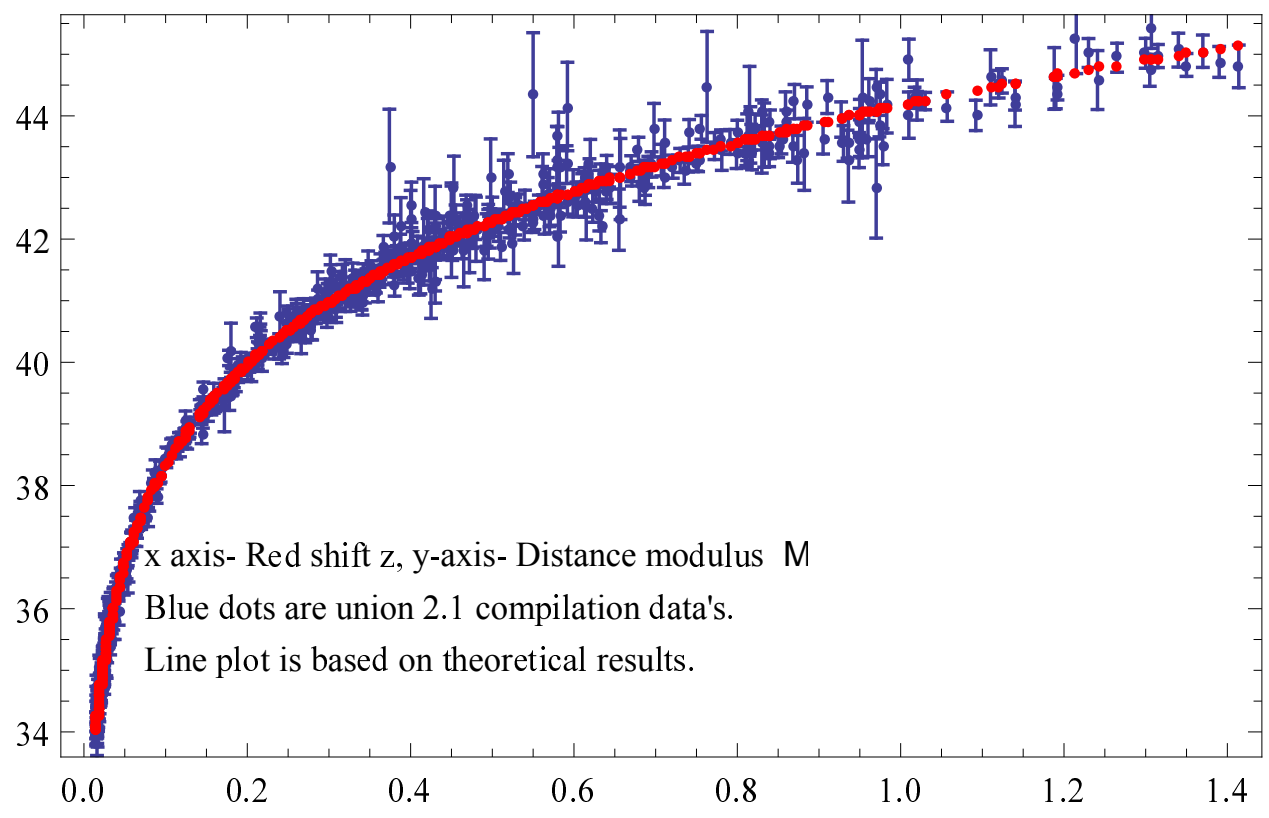

Figure 8: Plot of distance modulus $\left(\mu=M-m_{b}\right)$ versus redshift $(z)$ 
In the literature there are so many supernova of low red shift whose apparent magnitudes are known. These determines common absolute magnitude $M$ of all SN Ia supernovae. In our earlier work [43, 44], we have obtained $M$ as follows $M=5 \log _{10}\left(\frac{H_{0}}{.026 c}\right)-8.92$. From these, we obtain, $\log _{10}\left(H_{0} D_{L}\right)=\left(m_{b}-16.08\right) / 5+\log _{10}(.026 c)$ and following expression for apparent magnitudes

$$
m_{b}=16.08+5 \log _{10}\left[\frac{1+z}{.026} \int_{0}^{z} \frac{d z}{h(z)}\right] .
$$

Solving Eqs. (7) - (91) and (11) numerically, we present the following table describing the values of the apparent magnitude $m_{b}$ for redshift $z$ ranging between 0 and 1.6

[Table-7]

(The values of apparent magnitude $\left(m_{b}\right)$ for redshift $(z)$ in the range 0 to 1.6 )

\begin{tabular}{|c|c|c|c|c|c|c|c|c|c|}
\hline$z$ & 0.1 & 0.2 & 0.3 & 0.4 & 0.5 & 0.6 & 0.7 & 0.8 \\
\hline$m_{b}$ & 19.1639 & 20.8097 & 21.8154 & 22.5523 & 23.1379 & 23.6251 & 24.0426 & 24.4079 \\
\hline \hline$z$ & 0.9 & 1. & 1.1 & 1.2 & 1.3 & 1.4 & 1.5 & 1.6 \\
\hline$m_{b}$ & 24.7323 & 25.0239 & 25.2883 & 25.53 & 25.7523 & 25.958 & 26.149 & 26.3272 \\
\hline
\end{tabular}

We consider 581 data points of the observed values of the distance modulus of various SNe Ia type supernovas from the union 2.1 compilation [14] with red shift in the range $z \leq 1.414$. We calculate the corresponding theoretical values as per our model. The following Figures $7 \& 8$ depict the closeness of observational and theoretical results, thereby justifying our model.

\section{Conclusions}

In this work, efforts are made to develop a cosmological model which satisfies the cosmological principle and incorporates the latest development which envisaged that our universe is accelerating due to dark energy. We have also proposed a variable equation of state for dark energy in our model. We studied a model with radiation, dust and dark energy which shows a transition from deceleration to acceleration. We have successfully subjected our model to various observational tests.

In a nutshell, we believe that our study will pave the way to more research in future, in particular, in the area of the early universe, inflation and galaxy formation, etc. The proposed hybrid expansion law may help in the investigations of hidden matter like dark matter, dark energy and black holes.

\section{Acknowledgement}

The authors (G. K. Goswami \& A. Pradhan) sincerely acknowledge the Inter-University Centre for Astronomy and Astrophysics (IUCAA), Pune, India for providing facility where part of this work was completed during a visit. A. Pradhan would also like to thank the University of Zululand, South Africa for providing facilities and support where part of this work has been done. The authors would like to convey their sincere thanks to Sergei D. Odintsov ,Kazuharu Bamba and Sunny Vagnozzi for useful suggestions and providing references [45] - 52] for the improvement of the paper in present form. 


\section{References}

[1] Perlmutter, S. et al., 1998. Discovery of a supernova explosion at half the age of the Universe, Nature 391, 51.

[2] Perlmutter, S. et al., 1999. Measurements of $\Omega$ and $\Lambda$ from 42 high-redshift supernovae, Astrophys. J. 517, 5.

[3] Riess, A.G. et al., 1998. Observational evidence from supernovae for an accelerating universe and a cosmological constant, Astron. J. 116, 1009.

[4] Tonry, J.L. et al., 2003. Cosmological results from high-z supernovae, Astrophys. J. $594,1$.

[5] Clocchiatti, A. et al., 2006. Hubble Space Telescope and ground-based observations of type Ia Supernovae at redshift 0.5: cosmological implications, Astrophys. J. 642, 1.

[6] de Bernardis, P. et al., 2000. A flat universe from high-resolution maps of the cosmic microwave background radiation, Nature 404, 955-959.

[7] Hanany, S. et al., 2000. MAXIMA-1: a measurement of the cosmic microwave background anisotropy on angular scales of $10^{\prime}-5$, Astrophys. J. 545, L5-L9.

[8] Spergel, D.N. et al. [WMAP collaboration], 2003. First year wilkinson microwave anisotropy probe (WMAP) observations determination of cosmological parameters, Astrophys. J. Suppl. 148, 175.

[9] Tegmark, M. et al. [SDSS collaboration], 2004. Cosmological parameters from SDSS and WMAP, Phys. Rev. D 69, 103501.

[10] Seljak, U. et al., 2005. Cosmological parameter analysis including SDSS Ly $\alpha$ forest and galaxy bias constraints on the primordial spectrum of fluctuations neutrino mass and dark energy, Phys. Rev. D 2005, 71.

[11] Adelman-McCarthy, J. K. et al., 2006. The fourth data release of the sloan digital sky survey, Astrophys. J. Suppl. 162, 38.

[12] Bennett, C.L. et al., 2003. First year wilkinson microwave anisotropy probe (WMAP) observations preliminary maps and basic results, The Astrophys. J. Suppl. 148, 1-43.

[13] Allen, S.W. et al., 2004. Constraints on dark energy from chandra observations of the largest relaxed galaxy clusters, Mon. Not. R. Astron. Soc. 353, 457.

[14] Suzuki, N. et al., 2012. The Hubble space telescope cluster supernova survey $V$ improving the dark-energy constraints above $z>1$ and building an early-type-hosted supernova sample, Astrophys. J. 746, 85-115.

[15] Delubac, T. et al. [BOSS Collaboration], 2015. Baryon acoustic oscillations in the Ly $\alpha$ forest of BOSS DR11 quasars, Astron. Astrophys. 574, A59.

[16] Blake, C. et al. [The WiggleZ Dark Energy Survey], 2012. The wiggleZ dark energy survey joint measurements of the expansion and growth history at $z<1$, Mon. Not. R. Astron. Soc. 425, 405-414. 
[17] Ade, P.A.R. et al. [Planck Collaboration], 2016. Planck 2015 results XIV dark energy and modified gravity, Astron. Astrophys. 594, A14.

[18] Copeland, E.J. at el., Dynamics of dark energy, Int. J. Mod. Phys. D 15, 1753-1935.

[19] Grøn, Ø., Hervik, S. 2007. Einstein's general theory of relativity with modern applications in cosmology (Springer Publication ).

[20] Weinberg, S., 1989. The cosmological constant problem, Rev. Mod. Phys. 61, 1.

[21] Carroll, S.M., Hoffman, M., 2003. Can the dark energy equation-of-state parameter $\omega$ be less than -1, Phys. Rev. D 68, 023509.

[22] Riess, A.G. et al., 2004. Type Ia supernova discoveries at $z>1$ from the hubble space telescope: Evidence for past deceleration and constraints on dark energy evolution, Astrophys. J. 607, 665.

[23] Astier, P. et al., 2006. The supernova legacy survey measurement of $\Omega_{m}$, $\Omega_{\Lambda}$ and $\omega$ from the first year data set, Astron. Astrophys. 447, 31.

[24] Eisentein, D.J. et al., 2005. Detection of the baryon acoustic peak in the large-scale correlation function of SDSS luminous red galaxies, Astrophys. J. 633, 560.

[25] MacTavish, C.J. et al., 2006. Cosmological parameters from the 2003 flight of BOOMERANG, Astrophys. J. 647, 799.

[26] Komatsu, E. et al., 2009. Five-year wilkinson microwave anisotropy probe observations: Likelihoods and parameters from the wmap data, Astrophys. J. Suppl. Ser. 180, 330.

[27] Knop, R.K. et al., 2003. New constraints on $\Omega_{m}, \Omega_{\Lambda}$ and $\omega$ from an independent set of 11 high-redshift supernovae observed with the hubble space telescope, Astrophys. J. $598,102$.

[28] Hinshaw, G. et al., 2009. Nine-year wilkinson microwave anisotropy probe (WMAP) observations cosmological parameter results, Astrophys. J. Suppl. 180, 225.

[29] Amirhashchi, H., Pradhan, A., Saha, B., 2011. An interacting two-fluid scenario for dark energy in an FRW universe, Chin. Phys. Lett. 28, 039801.

[30] Amirhashchi, H., Pradhan, A., Zainuddin, H., 2011. An interacting and non-interacting two-fluid dark energy models in FRW universe with time dependent deceleration parameter, Int. J. Theor. Phys. 50, 3529.

[31] Pradhan, A., Amirhashchi, H., Saha, B., 2011. An interacting and non-interacting twofluid scenario for dark energy in FRW universe with constant deceleration parameter, Astrophys. Space Sci. 333, 343.

[32] Saha, B., Amirhashch, H., Pradhan, A., 2012. Two-fluid scenario for dark energy models in an FRW universe-revisited, Astrophys. Space Sci. 342, 257

[33] Pradhan, A., 2014. Two-fluid atmosphere from decelerating to accelerating FriedmannRobertsonWalker dark energy models, Indian J. Phys. 88, 215. 
[34] Kumar, S., 2011. Some FRW models of accelerating universe with dark energy, Astrophys. Space Sci. 332, 449.

[35] Goswami, G.K., Pradhan, A., Beesham, A., 2019. FLRW Accelerating Universe with Interactive Dark Energy, arXiv: 1906.00450.

[36] Zhang, C. et al., 2014. Four new observational $H(z)$ data from luminous red galaxies in the sloan digital sky survey data release seven, Res. Astron. Astrophys. 14, 1221-1233.

[37] Stern, D. et al., 2010. Cosmic chronometers constraining the equation of state of dark energy I $H(z)$ measurements, Jour. Cosmo. Astropart. Phys. 02, 008:

[38] Moresco, M., 2015. Raising the bar new constraints on the hubble parameter with cosmic chronometers at $z \sim 2$, Mon. Not. R. Astron. Soc. 450, L16-L20.

[39] Simon, J. et al., 2005. Constraints on the redshift dependence of the dark energy potential, Phys. Rev. D 71, 123001.

[40] Benitez, N. et al., 2002. The magnification of SN 1997ff the farthest known supernova, Astrophys. J. 577, L1.

[41] Turner, M., Riess, A.G., 2002. Do type Ia supernovae provide direct evidence for past deceleration of the universe?, Astrophys. J. 569, 18.

[42] Liddle, A. R., Lyth, D.H., 2000. Cosmological inflation and large-scale structure (Cambridge University Press).

[43] Goswami, G.K., Dewangan, R.N., Yadav, A.K., 2016. Anisotropic universe with magnetized dark energy, Astrophys. Space Sci. 361, 119.

[44] Goswami, G.K., Dewangan, R. N., Yadav, A. K., Pradhan, A., 2016. Anisotropic string cosmological models in Heckmann-Schucking space-time, Astrophys. Space Sci. 361, 47.

[45] Capozziello S., Luongo O., Saridakis E.N., 2015. Transition red shift in $\mathrm{f}(\mathrm{T})$ cosmology and observational constraints, Phys. Rev. D, 91, 12, 124037. .

[46] S. Capozziello S., Farooq O., Luongo O., Ratra B., 2014. Cosmographic bounds on the cosmological deceleration-acceleration transition redshift in $\mathrm{f}(\mathrm{R})$ gravity, Phys. Rev. D, 90, 4, 044016.

[47] Bamba K., Capozziello S., Nojiri S. and Odintsov S. D.,2012. General review of fluid DEs and its equivalence with scalar DE is given in Dark energy cosmology: the equivalent description via different theoretical models and cosmography tests, Astrophys. Space Sci.,342, 155 .

[48] Sunny Vagnozzi et al., 2018. Constraints on the sum of the neutrino masses in dynamical dark energy models with $\omega(z) \geq-1$ are tighter than those obtained in $\Lambda$ CDM, Phys. Rev. D 98 , 083501

[49] Capozziello S. et al., 2006. Observational constraints on dark energy with generalized equations of state, Phys.Rev. D 73, 043512 
[50] Nojiri S. and Odintsov S. D, 2004. The Final state and thermodynamics of dark energy universe, Phys.Rev. D70, 103522

[51] Nojiri S. and Odintsov S. D, 2006. The New form of the equation of state for dark energy fluid and accelerating universe, Phys.Lett. B 639,144-150.

[52] Nojiri S. and Odintsov S. D, 2006. Holographic DEs with phantom-non-phantom transitions were introduced in. Unifying phantom inflation with late-time acceleration: Scalar phantom-non-phantom transition model and generalized holographic dark energy, Gen.Rel.Grav. 38, 1285-1304. 